\title{
Lab on a Chick: A Novel In Vivo Angiogenesis Assay
}

Shanna Arnold*, Amanda Hansen*, Will Ashby*, David Schaffer**, Trenis Palmer*, Erik Olsen*, John Wikswo**, and Andries Zijlstra*

\footnotetext{
*Department of Pathology and Cancer Biology, Vanderbilt University, Nashville, TN 37232

** Vanderbilt Institute of Integrative Biosystems Research and Education, Vanderbilt University, Nashville, TN
}

While experimental cancer models have provided strong evidence that tumor growth and metastasis is dependent on neovascularization, the clinical application of angiogenesis inhibitors has shown minimal effect on tumor response and/or patient survival. The sub-optimal performance of angiogenesis inhibitors is due, in part, to the limitations of current angiogenesis assays. Most angiogenesis assays are performed in vitro for ease, cost reduction and the ability to quantify. However, in vitro assays fail to recapitulate conditions observed in the complex tumor microenvironment. Several in vivo and ex vivo angiogenesis assays exist that allow the study of neovascularization within the tumor microenvironment but are costly as far as the use of mouse models, the amount of drug required for therapeutic effect and the lengthy experimental time-frame. We have developed a novel in vivo angiogenesis assay that reduces time, cost and reagent usage while allowing quantification and intravital imaging.

To obtain a clear evaluation of tumor-induced angiogenesis we generated a bioreactor for a well-established angiogenesis model, the chorioallantoic membrane (CAM). We were able to evaluate neovascularization specifically in response to the tumor cells by creating a fixed spatial separation of the host and tumor. The "Lab on a Chick" bioreactor (angiogenesis disk) was constructed as a circle with four inner chambers having $20 \mu \mathrm{m}$ perforations. Each inner chamber is connected to an outer chamber through a single channel. The outer chambers lack perforations. Tumor cells are embedded in collagen in the outer chamber while collagen alone is added to the inner chamber. The angiogenesis disk is then grafted directly on top of the chorioallantoic membrane (CAM) of an ex ovo (eggless) chicken embryo. In this manner, only the inner chamber is in contact with the CAM through the perforations. The tumor cells are free to migrate into the inner chamber and tumor secreted factors can diffuse across the pores and into the CAM, thus stimulating angiogenesis. Derivations of this assay are possible by altering the embedding matrix and/or incorporating therapies directly into the disk chambers. Furthermore, the angiogenesis disks can have a hypoxic or normoxic environment depending on whether glass or pdms is used to coverslip the disk.

We demonstrate that tumor-induced angiogenesis occurs in response to the adjacent tumor cells. Patent vasculature forms within and around micro-tumors in the angiogenesis disk after as little as four days. Furthermore, manipulation of oxygen levels and/or the addition of angiogenesis inhibitors alter the vascular response within the grafted disk. In addition, tumor cell invasion in response to hypoxia and anti-angiogenic therapy can be monitored. Lastly, we demonstrate that our in vivo angiogenesis assay allows for the monitoring of neovascularization and tumor cell motility in real-time. 

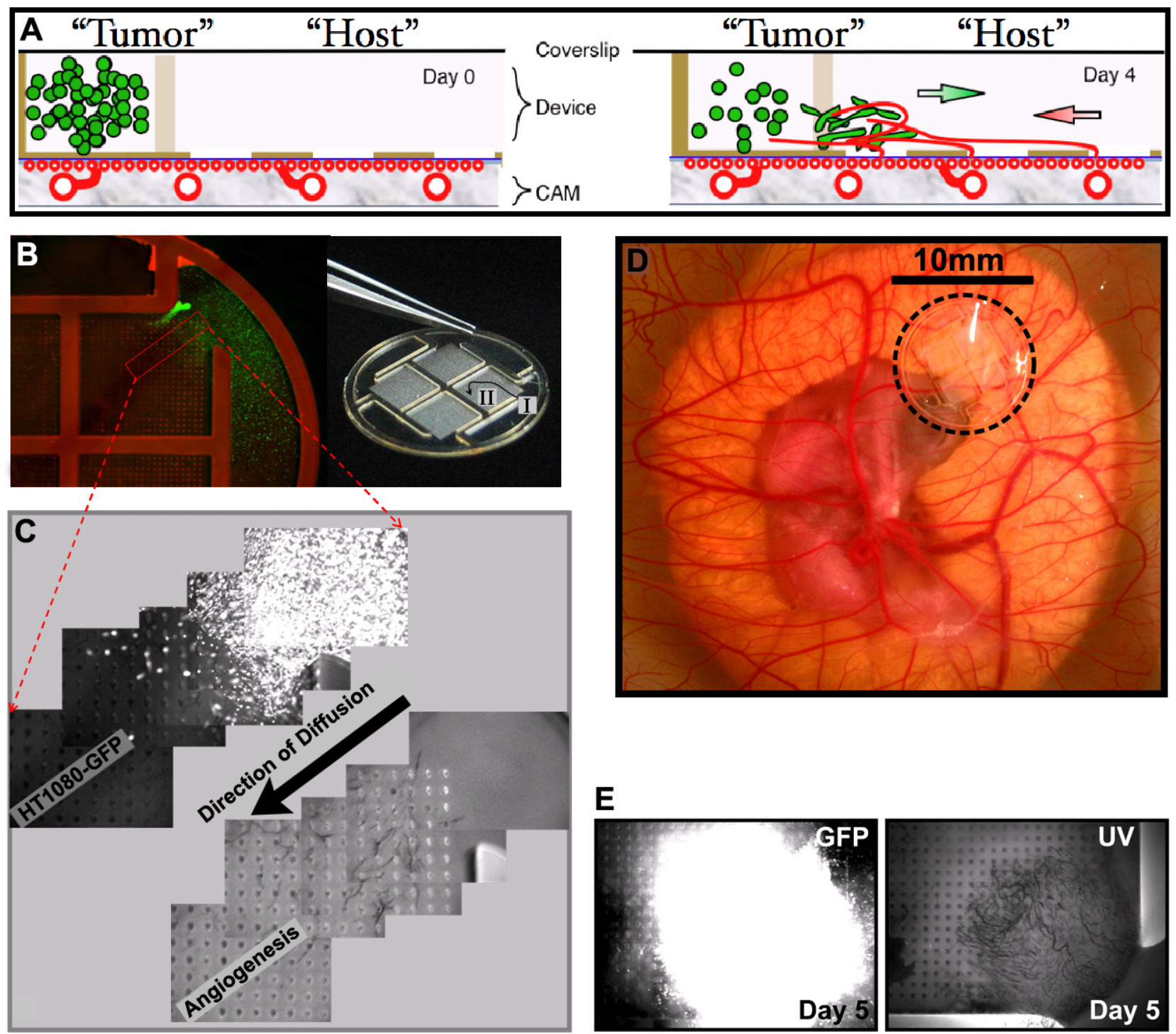

Figure 2. Angiogenesis Disk A) Schematic representation of the reciprocal tumor invasion and angiogenesis. Tumor cells exit the outer chamber through a small opening that provides access to the internal quadrant. This quadrant is in contact with the CAM through perforations in the bottom which allow the CAM vasculature to invade the device. B) The angiogenesis disk is a non-toxic epoxy based photoresist, SU-8. Inner quadrants (II) contain $20 \mu \mathrm{m}$ perforations while the outer chambers (I), where the tumor cells are seeded, are devoid of contact with the CAM. C) Host vasculature forms in response to tumor cells along a concentration gradient. D) Angiogenesis and tumor cell invasion can be imaged in real time utilizing the "eggless" chick model. Disks are 10mm in diameter and are placed directly onto the chick chorioallantoic membrane (CAM). This allows in vivo imaging and monitoring of angiogenesis and invasion without invasive procedures. E) Proof of principle: Angiogenesis disks were constructed with $1.5 \mathrm{mg} / \mathrm{ml}$ of collagen I and outer chambers were loaded with 50,000 cells per chamber of HEp3-GFP cells. The entire disk was placed on the CAM and cover-slipped with an 80-100 $\mu$ m film of PDMS. Image of HEp3-GFP cells (GFP) within the inner quadrant of the disk taken 5 days after placement on the CAM and the corresponding patent neovasculature (UV). Images taken at $45 \mathrm{X}$ total magnification. 